\title{
Radiosensitivity Index Predicts for Survival with Adjuvant Radiation in Resectable Pancreatic Cancer
}

Running title: RSI Predictive in Pancreatic Cancer

Tobin Strom MD ${ }^{1}$, Sarah E. Hoffe MD ${ }^{1}$, William Fulp, $\mathrm{MS}^{3}$, Jessica Frakes MD ${ }^{1}$, Domenico Coppola $\mathrm{MD}^{2}$, Gregory M. Springett MD-PhD², Mokenge P. Malafa MD², Cynthia L Harris, $\mathrm{MD}^{2}$, Steven A Eschrich, $\mathrm{PhD}^{3}$, Javier F. Torres-Roca, $\mathrm{MD}^{1}$, and Ravi Shridhar, MD-PhD ${ }^{1}$

${ }^{1}$ Department of Radiation Oncology, H. Lee Moffitt Cancer Center and Research Institute, Tampa, FL ${ }^{2}$ Gastrointestinal Tumor Program, H. Lee Moffitt Cancer Center and Research Institute, Tampa, FL ${ }^{3}$ Department of Biomedical Informatics, H. Lee Moffitt Cancer Center and Research Institute, Tampa, FL

Keywords: pancreas; cancer; radiation; radiosensitivity; surgery; survival

Pages: 13 Tables: $2 \quad$ Figures: 3

\section{Corresponding authors:}

Ravi Shridhar, M.D., Ph.D., Javier F. Torres-Roca, M.D.

Department of Radiation Oncology

H. Lee Moffitt Cancer Center and Research Institute

12902 Magnolia Drive

Tampa, FL 33612, U.S.A.

Email: Ravi.Shridhar@moffitt.org, Javier.TorresRoca@moffitt.org

Office Phone: 00-1-813-745-1824

Office Fax: 00-1-813-745-7231 


\begin{abstract}
Background and Purpose: Adjuvant radiation therapy for resectable pancreatic cancer remains controversial. Sub-populations of radiosensitive tumors might exist given the genetic heterogeneity of pancreatic cancers. We evaluated whether RSI is predictive of survival in pancreatic cancer treated with radiation.
\end{abstract}

Materials and Methods: We identified 73 genomically-profiled pancreas cancer patients treated with upfront surgery between 2000 and 2011 (48 radiation, 25 no radiation). Briefly, RSI score is derived from the expression of 10 specific genes and a linear regression algorithm modeled on SF2 of 48 cancer cells. The primary endpoint was to assess the association of RSI with overall survival.

Results: Median follow-up was 67 months for surviving patients. On multivariate analysis, patients with radioresistant tumors had a trend toward worse survival (Hazard ratio [HR] 2.1[95\%CI 1.0-4.3], $\mathrm{p}=0.054$ ). Among high-risk, irradiated patients (positive margins, positive lymph nodes, or a postoperative CA 19-9>90; $n=31$ ), radiosensitive patients had significantly improved survival compared with radioresistant patients (median 31.2 vs. 13.2 months; HR 0.42[0.19,0.94], p=0.04). Among irradiated patients $(n=48)$, low-risk patients lived longer than both high-risk patients with radiosensitive tumors and radioresistant tumors (HR 2.7[1.0,7.2], $\mathrm{p}=0.04$ and HR 6.3[2.3,17.0], $\mathrm{p}<0.001$, respectively). Conclusions: Integrating RSI with standard high-risk variables has the potential to refine the classification of high-risk resected pancreatic cancer patients treated with radiation therapy. 


\section{Introduction}

Pancreatic cancer is the $4^{\text {th }}$ most common cause of cancer mortality in the United States, and will account for an estimated 45,220 new cases and 38,460 deaths in the United States in 2013.[1] Patients with pancreatic cancer have a poor prognosis, with only $20 \%$ of patients presenting with potentially curable, local disease at initial diagnosis, and 5 year outcomes among all patients ranging from 5-6\%.[1, 2] Recent data from a rapid autopsy series suggests that there are genetic subtypes of pancreatic cancers associated with two distinct patterns of failure, either locally destructive or metastatic, with DPC4 gene status correlating with widespread metastasis.[3]

Adjuvant radiation therapy (RT) for resectable pancreatic cancer remains controversial and has not been clearly shown to improve outcomes in randomized phase III trials[4, 5]. The lack of definitive survival benefit with adjuvant RT or chemoradiation therapy (CRT) might result from the wide heterogeneity of genetic changes present in pancreatic cancers, leading to high rates of radioresistance.[6, 7] One study using DNA sequencing and microarray analysis reported an average of 63 genetic mutations present per pancreatic cancer as measured from 24 pancreatic tumors.[6] Recent studies support that cancer stem cells likely play a key role in intratumoral cellular heterogeneity which can lead to chemotherapy and radiotherapy resistance.[8] With respect to pancreatic cancer, pancreatic stellate cells have been shown to enhance the cancer stem cell phenotype and to promote tumor progression and radioresistance.[9] Given the wide variety of genetic mutations and the inherent radioresistance of pancreatic cancer, as well as the paucity of tools to help guide adjuvant RT recommendations, a genomic signature addressing pancreatic tumor radiosensitivity could substantially improve our decision-making process for, or against, adjuvant RT.

In previous studies we developed RSI, a genomic signature specific for tumor radiosensitivity that has been clinically validated as an RT-specific biomarker in multiple disease sites.[10,11] The purpose of this study was to evaluate whether RSI is predictive for survival in pancreatic cancer treated with radiation. 


\section{Materials and Methods}

\section{Patient Characteristics}

Patients with pancreatic cancer were identified from an IRB-approved prospective observational protocol at our institution. The Total Cancer Care (TCC) initiative includes a comprehensive patient database and tissue bank from 17 cancer centers around the nation.[12] In order to assess whether RSI is predictive in patients with resectable pancreatic cancer treated with radiation, we used the TCC to identify patient, tumor, and treatment characteristics for all patients treated with resectable pancreatic cancer. We restricted our analysis to patients who underwent upfront surgical resection for pancreatic cancer between 2000 and 2011 and who had tissue samples available for analysis (Figure 1, n=73). Patients were excluded if they had non-carcinoma histologies, incomplete patient records, metastatic disease at presentation, or were treated with neoadjuvant therapy including chemotherapy and/or radiation therapy. Patients who lived less than 90 days after surgery were also excluded since these patients were not offered and/or treated with radiation therapy. All patients with biopsy-proven pancreatic cancer underwent a preoperative staging work-up including an endoscopic ultrasound, a computed tomography scan with intravenous contrast of the thorax, abdomen and pelvis \pm magnetic resonance imaging \pm a positron emission tomography scan to determine resectability. All cases were presented at the gastrointestinal tumor board to determine resectability.

\section{Surgery}

Patients with pancreatic head tumors underwent pancreaticoduodenectomy with or without a pylorus-sparing procedure. A minority of patients with pancreatic body or tail tumors underwent pancreaticoduodenectomy, complete pancreatectomy, or partial pancreatectomy with or without splenectomy. All but 4 patients underwent regional lymph node sampling or dissection. Post-operative CA19-9 levels were recorded in $76 \%$ of resectable pancreatic cancer cases.

\section{Adjuvant therapy}


Following surgery, patients received one of three treatment schemas: chemoradiation $(n=48)$ with or without adjuvant chemotherapy, chemotherapy alone $(n=13)$, or no adjuvant therapy $(n=12)$. Adjuvant therapy was initiated within 6 months from the time of surgery in all cases.

Patients treated with chemotherapy alone received single-agent gemcitabine. The majority of patients treated with chemotherapy followed by chemoradiation $(n=21)$ were treated in a similar fashion to the RTOG 9704 protocol with 1 month of gemcitabine followed by concurrent chemoradiation with continuous infusion 5-FU followed by adjuvant gemcitabine. The median total radiation dose was 50.4 Gy (range 45-54 Gy) in 180 to 200 cGy daily fractions for a median of 28 fractions (range 24-30) to the pancreatic tumor bed and regional lymphatics; a minority of patients received a boost to the tumor bed (median 5.4 Gy, range 1.8-9 Gy). Seven patients had an unknown radiation dose.

\section{Radiosensitivity Index}

Beginning in 2006, all available fresh-frozen tumor tissues in the TCC tissue bank were profiled for gene expression (Affymetrix Hu-RSTA-2a520709).[13] Using the gene micro-array data, an RSI score (range 0-1.0) was derived from the expression of 10 specific genes and a linear regression algorithm modeled on SF2 of 48 cancer cells as previously described by Eschrich et al.[10] RSI-high (radioresistant) and RSI-low (radiosensitive) groups were split at the median-RSI point of the radiation therapy group $(n=48)$ for analysis and rounded to the nearest $1 / 100^{\text {th }}$ RSI value $(R S I=0.36)$. That left 25 patients in the radioresistant group and 23 in the radiosensitive group. The same RSI cut-point was then used to split the patients not treated with adjuvant radiation therapy $(n=25)$.

\section{Endpoints and Statistical Analysis}

The primary endpoint of the study was to assess the association of RSI with overall survival in patients with resectable pancreatic cancer who did and did not receive adjuvant radiation therapy. Overall survival was defined as the interval from surgery to date of death.

Statistical analysis was performed using $\operatorname{SPSS}^{\circledR}$ version $22.0\left(\right.$ IBM $^{\circledR}$, Chicago, IL). Continuous variables were compared using both Wilcoxon rank sum test. Pearson's Chi-square test was used to compare categorical variables. Actuarial rates of overall survival were calculated using the Kaplan-Meier 
method and the log-rank test. A Cox multivariate model was performed for overall survival, including receipt of adjuvant radiation therapy, RSI groupings, total radiation dose grouped by $45 \mathrm{~Gy}(\mathrm{n}=9), 50$ 50.4 Gy (n=27), >50.4 Gy (n=5) and unknown RT dose $(\mathrm{n}=7)$, and a single clinicopathologic high-risk variable including patients with positive surgical margins, positive lymph nodes on pathology, and/or a post-operative CA 19-9>90 ng/mL. Final multivariate models were determined using Akaike Information Criterion. All statistical tests were two-sided and an $\alpha$ (type I) error $<0.05$ was considered statistically significant.

\section{Results}

We identified 73 patients with resectable pancreatic cancer who underwent upfront surgery with both RSI and clinical outcome available (Figure 1; 48 RT, 25 no RT). The median follow-up among surviving patients was 67 months (range 25-141). Patient and tumor characteristics with and without RT are presented in Table 1. Patients treated with adjuvant radiation therapy were significantly younger than patients treated without radiation therapy (median age 64 vs. 74 years, respectively; $p<0.001$ ), but otherwise there was no was no difference between the groups. Patient characteristics separated by RSI group are also presented in Table 1; when split by RSI, there was no significant difference in presenting patient or tumor characteristics.

Among all patients $(n=73)$, only high-risk pancreatic patients, including those with positive surgical margins, involved pathologic lymph nodes, or a post-operative CA $19-9>90 \mathrm{ng} / \mathrm{mL}$, had significantly worse survival on multivariate analysis compared low-risk patients (Figure 2a, median not reached vs. 18.8 months, respectively; Table 2 , HR $2.3[1.2,4.4], \mathrm{p}=0.01)$. This confirms the prognostic value of the high-risk group. In contrast, adjuvant radiation therapy and RSI were not associated with survival among all patients.

In the subgroup of patients treated with radiation therapy $(n=48)$, median survival for patients with radiosensitive tumors was 32.1 months compared with 18.3 months for patients with radioresistant 
tumors (Figure $2 b, p=0.45$ ). On multivariate analysis, patients with radioresistant tumors had a trend toward worse survival (Table 2, HR 2.1 [1.0,4.3], $\mathrm{p}=0.054$ ). Radiation dose was not associated with survival ( $p>0.05$ ). Among clinicopathologic low-risk, irradiated pancreatic patients, there was no difference in survival by RSI status (log-rank p=0.9). However, among clinicopathologic high-risk, irradiated patients, radiosensitive patients had significantly longer survival than radioresistant patients (median 31.2 vs. 13.2 months, respectively; HR: 2.4 [1.1,5.4], p=0.04). When low-risk patients were compared with high-risk radiosensitive and high-risk radioresistant patients, they had significantly longer survival than both high-risk patient populations (Figure 3 and Supplementary Table, 3-year overall survival $65 \%$ vs. $41 \%$ vs. $7 \%$, respectively; HR: 2.7 [1.0,7.2], p=0.04 and HR: 6.3 [2.3,17.0], respectively, $\mathrm{p}<0.001)$.

\section{Discussion}

This is the first study to demonstrate that a radiation-specific stratification method is feasible among high-risk resectable pancreatic cancer patients treated with radiation therapy. The RSI score has previously been validated in six independent, prospectively collected data sets, including esophageal, rectal, head and neck, breast, and colon cancer patients.[10, 11, 14] The RSI score is not specific to pancreatic cancer, but includes genes that affect DNA damage response, histone deacetylation, the cell cycle, apoptosis and cellular proliferation.[11] A unique benefit of the RSI score is that it has the potential to address the role of radiation therapy across many different oncologic settings. Most genomic signatures in clinical use have been developed as disease site-specific predictors of recurrence risk (i.e. OncotypeDX (breast), Mammaprint (breast), Prolaris (prostate) etc).[15-19] In contrast, the RSI score has been shown to be useful in multiple settings with radiation therapy. In the current study, we demonstrate a

possible role for the RSI as a supplement to known clinicopathologic high-risk factors to predict survival in patients with pancreatic cancer.

The data for, or against, adjuvant radiation therapy in the resectable pancreatic cancer setting is lacking. As a result, there is controversy as to whether radiation therapy truly is beneficial.[20] There are a few possible explanations as to why radiation therapy has not been clearly shown to benefit patients 
with resectable pancreatic cancer. Previous prospective trials addressing adjuvant radiation therapy in pancreatic cancer have been flawed by low patient numbers (GITSG 9173[21]), poor study design (ESPAC-1[22, 23]), out-dated radiation doses and split-course radiation regimens (GITSG 9173, EORTC 40891, ESPAC-1) and poor quality control and administration of radiation therapy (ESPAC-1[22, 23]). A recent analysis of RTOG 9704 demonstrated significantly improved survival among patients treated with radiation therapy per protocol.[24] This suggests that radiation targeting and delivery is extremely important in pancreatic cancer outcomes and the lack of radiation quality control in previous prospective, randomized trials possibly played a role in the inability to demonstrate a clear benefit with adjuvant radiation. Indeed, a study addressing radiation targeting and tumor motion during radiation therapy for locally advanced pancreatic cancer have found that commonly used CTV-PTV margins inadequately account for target motion during pancreatic radiotherapy. As a result, patient mobilization, accounting for respiratory motion and 4D planning are beneficial for radiotherapy planning.[25] This may play a role in post-operative setting as well, where pre-operative imaging can be used as a guide for the volume delineation. Radiation modality might also play a role in outcomes, as pre-clinical data has shown a pancreatic cell death advantage with the use of carbon ion therapy compared with photons.[26] An additional factor that might contribute to the lack of benefit with radiation therapy is that pancreatic cancers are relatively radioresistant, and as a result, residual tumor cells following surgery are likely less affected by radiation.[9] The cancer stem cell hypothesis supports this notion, with increased intratumoral cellular heterogeneity leading to increased radioresistance. In-vivo pancreatic tumor models have shown that pancreatic stellate cells enhance the epithelial to mesenchymal transition and play a key role in the cancer stem cell process for pancreatic cancer. As a result, molecular targets that interfere with this pathway, such as TGF $\beta$, have been shown to increase the radiosensitivity of pancreatic tumor cells.[8] Increasing the radiosensitivity of pancreatic cancer cells is a promising method of improving outcomes. Another possible method of improving outcomes is to identify sub-populations of patients who have pancreatic tumors with inherently radiosensitive tumors. In the current study, we present data that supports this possibility. 
Although the classic prognostic markers including CA19-9, surgical margins status and lymph node status were able to distinguish two prognostic classes with different clinical outcomes, the addition of RSI among irradiated patients revealed three distinct groups with a wide distribution of clinical outcome (Supplementary Table, 3 year overall survival 65\% vs. 41\% vs. 7\%, for low-risk, high-risk radiosensitive and high-risk radioresistant groups, respectively, $\mathrm{p}<0.001)$. In addition, RSI only added predictive information in patients treated with radiation, consistent with its role as a radiosensitivity biomarker, although we acknowledge that these conclusions are limited by the small number of patients in both patients who did, and did not, receive radiation. The cohort who did not receive RT was not large enough to make a definite conclusion regarding the predictive value of RSI and we cannot exclude that RSI is prognostic rather than predictive. Thus, we propose that RSI used in patients with positive surgical margins, involved pathologic lymph nodes, or a post-operative CA 19-9 >90 ng/mL, could be a useful tool to help guide physicians with their recommendations for or against adjuvant radiation therapy.

To be clear, RSI was developed as radiation-specific biomarker. Both gene selection and algorithm development were exclusively based on in vitro SF2 for 48 cancer cell lines. We have previously demonstrated that RSI only predicts outcome in RT-treated patients.[10, 11] Thus, as a radiosensitivity biomarker, RSI can only predict clinical outcomes in patient populations that benefit from radiation therapy. Since the therapeutic benefit of radiation therapy in pancreas cancer remains controversial, the potential for a radiosensitivity biomarker in this disease is expected to be limited. In addition, radiation therapy as an individual predictive marker among all irradiated patients did not demonstrate a clear overall survival benefit in the current study $(\mathrm{p}=0.054)$. Consistent with this, RSI score as a solitary, binary, variable did not add predictive information on its own. However, when RSI was combined with the clinicopathologic high-risk patient population, two patient populations with significantly different outcomes emerged $(\mathrm{p}=0.04)$, suggesting that there are unrecognized subpopulations of patients who might benefit from adjuvant radiation therapy. Thus, we propose that analyses that incorporate both prognostic and predictive variables are better able to define this clinical sub-population heterogeneity. 
This study was limited by its small sample size and retrospective nature. There are also likely additional factors that influence the effect of radiation therapy in the pancreatic cancer setting. Hypoxia is not directly addressed by the RSI assay, yet pancreatic cancers have been shown to thrive in a hypovascular environment,[27] which has been reported to also increase the metastatic potential of solid tumors, which can lead to radioresistance.[28, 29] Since the RSI score is an estimate of radiosensitivity, it can only detect clinical differences if radiation therapy has an impact on the underlying cancer. The sample size of the cohort that did not receive radiation therapy was also small $(n=25)$, and as a result, few conclusions can be drawn from this group of patients. Despite this, the study did include a homogeneous and consistent population of patients with resectable pancreatic carcinoma, treated at a single-institution, with relatively consistent techniques among physicians from diagnosis to treatment.

In conclusion, integrating RSI with standard prognostic variables has potential to refine the classification of high-risk resected pancreatic cancer patients treated with radiation therapy. Prospective validation of RSI in patients with resectable pancreatic cancer is necessary. 


\section{References}

[1] Siegel R, Naishadham D, Jemal A. Cancer statistics, 2013. CA: a cancer journal for clinicians. 2013;63:11-30.

[2] Li D, Xie K, Wolff R, Abbruzzese JL. Pancreatic cancer. Lancet. 2004;363:1049-57.

[3] Iacobuzio-Donahue CA, Fu B, Yachida S, Luo M, Abe H, Henderson CM, et al. DPC4 gene status of the primary carcinoma correlates with patterns of failure in patients with pancreatic cancer. Journal of clinical oncology : official journal of the American Society of Clinical Oncology. 2009;27:1806-13.

[4] Neoptolemos JP, Stocken DD, Friess H, Bassi C, Dunn JA, Hickey H, et al. A randomized trial of chemoradiotherapy and chemotherapy after resection of pancreatic cancer. The New England journal of medicine. 2004;350:1200-10.

[5] Smeenk HG, van Eijck CH, Hop WC, Erdmann J, Tran KC, Debois M, et al. Long-term survival and metastatic pattern of pancreatic and periampullary cancer after adjuvant chemoradiation or observation: long-term results of EORTC trial 40891. Annals of surgery. 2007;246:734-40.

[6] Jones S, Zhang X, Parsons DW, Lin JC, Leary RJ, Angenendt P, et al. Core signaling pathways in human pancreatic cancers revealed by global genomic analyses. Science. 2008;321:1801-6.

[7] Hidalgo M. Pancreatic cancer. The New England journal of medicine. 2010;362:1605-17.

[8] Peitzsch C, Kurth I, Kunz-Schughart L, Baumann M, Dubrovska A. Discovery of the cancer stem cell related determinants of radioresistance. Radiotherapy and oncology : journal of the European Society for Therapeutic Radiology and Oncology. 2013;108:378-87.

[9] Al-Assar O, Demiciorglu F, Lunardi S, Gaspar-Carvalho MM, McKenna WG, Muschel RM, et al. Contextual regulation of pancreatic cancer stem cell phenotype and radioresistance by pancreatic stellate cells. Radiotherapy and oncology : journal of the European Society for Therapeutic Radiology and Oncology. 2014;111:243-51. 
[10] Eschrich SA, Pramana J, Zhang H, Zhao H, Boulware D, Lee JH, et al. A gene expression model of intrinsic tumor radiosensitivity: prediction of response and prognosis after chemoradiation. International journal of radiation oncology, biology, physics. 2009;75:489-96.

[11] Eschrich SA, Fulp WJ, Pawitan Y, Foekens JA, Smid M, Martens JW, et al. Validation of a radiosensitivity molecular signature in breast cancer. Clinical cancer research : an official journal of the American Association for Cancer Research. 2012;18:5134-43.

[12] Fenstermacher DA, Wenham RM, Rollison DE, Dalton WS. Implementing personalized medicine in a cancer center. Cancer journal. 2011;17:528-36.

[13] Welsh EA, Eschrich SA, Berglund AE, Fenstermacher DA. Iterative rank-order normalization of gene expression microarray data. BMC bioinformatics. 2013;14:153.

[14] Ahmed KA, Fulp WJ, Berglund AE, Hoffe SE, Dilling TJ, Eschrich SA, et al. Differences Between Colon Cancer Primaries and Metastases Using a Molecular Assay for Tumor Radiation Sensitivity Suggest Implications for Potential Oligometastatic SBRT Patient Selection. International journal of radiation oncology, biology, physics. 2015.

[15] van de Vijver MJ, He YD, van't Veer LJ, Dai H, Hart AA, Voskuil DW, et al. A gene-expression signature as a predictor of survival in breast cancer. The New England journal of medicine. 2002;347:1999-2009.

[16] van 't Veer LJ, Dai H, van de Vijver MJ, He YD, Hart AA, Mao M, et al. Gene expression profiling predicts clinical outcome of breast cancer. Nature. 2002;415:530-6.

[17] Paik S, Shak S, Tang G, Kim C, Baker J, Cronin M, et al. A multigene assay to predict recurrence of tamoxifen-treated, node-negative breast cancer. The New England journal of medicine. 2004;351:281726.

[18] Bishoff JT, Freedland SJ, Gerber L, Tennstedt P, Reid J, Welbourn W, et al. Prognostic utility of the cell cycle progression score generated from biopsy in men treated with prostatectomy. The Journal of urology. 2014;192:409-14. 
[19] Cuzick J, Swanson GP, Fisher G, Brothman AR, Berney DM, Reid JE, et al. Prognostic value of an RNA expression signature derived from cell cycle proliferation genes in patients with prostate cancer: a retrospective study. The Lancet Oncology. 2011;12:245-55.

[20] Katz MH, Landry J, Kindler HL. Current controversies in the stage-specific multidisciplinary management of pancreatic cancer. American Society of Clinical Oncology educational book / ASCO American Society of Clinical Oncology Meeting. 2014:e157-64.

[21] Kalser MH, Ellenberg SS. Pancreatic cancer. Adjuvant combined radiation and chemotherapy following curative resection. Archives of surgery. 1985;120:899-903.

[22] Choti MA. Adjuvant therapy for pancreatic cancer--the debate continues. The New England journal of medicine. 2004;350:1249-51.

[23] Koshy MC, Landry JC, Cavanaugh SX, Fuller CD, Willett CG, Abrams RA, et al. A challenge to the therapeutic nihilism of ESPAC-1. International journal of radiation oncology, biology, physics. 2005;61:965-6.

[24] Abrams RA, Winter KA, Regine WF, Safran H, Hoffman JP, Lustig R, et al. Failure to adhere to protocol specified radiation therapy guidelines was associated with decreased survival in RTOG 9704--a phase III trial of adjuvant chemotherapy and chemoradiotherapy for patients with resected adenocarcinoma of the pancreas. International journal of radiation oncology, biology, physics. 2012;82:809-16.

[25] Whitfield G, Jain P, Green M, Watkins G, Henry A, Stratford J, et al. Quantifying motion for pancreatic radiotherapy margin calculation. Radiotherapy and oncology : journal of the European Society for Therapeutic Radiology and Oncology. 2012;103:360-6.

[26] Oonishi K, Cui X, Hirakawa H, Fujimori A, Kamijo T, Yamada S, et al. Different effects of carbon ion beams and X-rays on clonogenic survival and DNA repair in human pancreatic cancer stem-like cells. Radiotherapy and oncology : journal of the European Society for Therapeutic Radiology and Oncology. 2012;105:258-65. 
[27] Feig C, Gopinathan A, Neesse A, Chan DS, Cook N, Tuveson DA. The pancreas cancer microenvironment. Clinical cancer research : an official journal of the American Association for Cancer Research. 2012;18:4266-76.

[28] Le QT, Denko NC, Giaccia AJ. Hypoxic gene expression and metastasis. Cancer metastasis reviews. 2004;23:293-310.

[29] Brown JM, Giaccia AJ. The unique physiology of solid tumors: opportunities (and problems) for cancer therapy. Cancer research. 1998;58:1408-16. 


\section{Figure Legends}

Figure 1: Flow chart demonstrating inclusion criteria for 73 evaluable patients who underwent upfront resection for pancreatic carcinoma with microarray and RSI data available.

Figure 2: Kaplan Meier overall survival plot (a) of all patients $(n=73)$ assessed by low risk (negative margins, negative lymph nodes, and a post-operative CA 19-9 $\leq 90$ ) vs. high-risk (positive margins, positive lymph nodes, or a post-operative CA 19-9 >90, p=0.002) and (b) of patients treated with radiation therapy $(\mathrm{N}=48)$ by (b) radiosensitivity index (RSI)-low (radiosensitive) vs. RSI-high (radioresistant, $\mathrm{p}=0.45$ ).

Figure 3. Kaplan Meier overall survival plot of patients treated with radiation therapy $(\mathrm{N}=48)$ assessed by clinicopathologic low-risk (negative margins, negative lymph nodes, and a post-operative CA $19-9 \leq 90$ ) vs. high-risk + radiosensitivity index (RSI)-low (radiosensitive) vs. high-risk + RSI-high (radioresistant, $\mathrm{p}<0.001)$ 
Table 1. Patient characteristics with, and without, radiation therapy (RT), and with radiosensitive (Radiosensitivity Index [RSI]-low) compared with radioresistant (RSI-high) patients. Patient age was the only difference between the clinicopathologic variables, with significantly younger patients in the irradiated group.

\begin{tabular}{|c|c|c|c|c|c|c|c|}
\hline Variable & & $\begin{array}{l}\text { RT; } \\
\text { n }(\%)\end{array}$ & $\begin{array}{l}\text { No RT; } \\
\text { n }(\%)\end{array}$ & $p$-value & $\begin{array}{c}\text { Radiosensitive; } \\
\text { n (\%) }\end{array}$ & $\begin{array}{c}\text { Radioresistant; } \\
\text { n (\%) }\end{array}$ & $p$-value \\
\hline Median Age (range) & & $64(25,81)$ & $74(43,87)$ & $<0.001$ & $68(38,86)$ & $69(25,87)$ & 0.20 \\
\hline \multirow[t]{2}{*}{ Sex } & Male & $27(56.3)$ & $14(56)$ & 0.98 & $14(45.2)$ & $27(64.3)$ & 0.10 \\
\hline & Female & $21(43.8)$ & $11(44)$ & & $17(54.8)$ & $15(35.7)$ & \\
\hline \multirow[t]{2}{*}{ Site } & Head & $39(81.3)$ & $19(76)$ & 0.60 & $26(83.9)$ & $32(76.2)$ & 0.42 \\
\hline & Body/Tail & $9(18.8)$ & $6(24)$ & & $5(16.1)$ & $10(23.8)$ & \\
\hline $\begin{array}{c}\text { Median Path Tumor size } \\
\text { (cm, range) }\end{array}$ & & $\begin{array}{c}3.0(0.8, \\
14.7)\end{array}$ & $\begin{array}{c}2.5(1.3 \\
8.0)\end{array}$ & 0.60 & $2.5(0.8,8.0)$ & $3.0(1.0,14.7)$ & 0.12 \\
\hline \multirow[t]{2}{*}{ Pathologic Tumor Stage } & $1 \& 2$ & $9(18.8)$ & $7(28)$ & 0.36 & $7(22.6)$ & $9(21.4)$ & 0.91 \\
\hline & $3 \& 4$ & $39(81.3)$ & $18(72)$ & & $24(77.4)$ & $33(78.6)$ & \\
\hline $\begin{array}{c}\text { Median Nodes Positive } \\
\text { (range) }\end{array}$ & & $1(0,9)$ & $0(0,12)$ & 0.85 & $1(0,9)$ & $1(0,12)$ & 0.88 \\
\hline $\begin{array}{c}\text { Median Nodes Removed } \\
\text { (Range) }\end{array}$ & & $12(1,43)$ & $14(2,49)$ & 0.15 & $12(1,49)$ & $13(1,43)$ & 0.56 \\
\hline \multirow[t]{2}{*}{ Pathologic Nodal Stage } & 0 & $20(41.7)$ & $14(56)$ & 0.24 & $13(41.9)$ & $21(50)$ & 0.49 \\
\hline & 1 & $28(58.3)$ & $11(44)$ & & $18(58.1)$ & $21(50)$ & \\
\hline \multirow[t]{2}{*}{ Known Tumor Grade } & Well/Moderate & $32(76.2)$ & $18(78.3)$ & 0.85 & $21(77.8)$ & $29(76.3)$ & 0.89 \\
\hline & Poor/Undifferentiated & $10(23.8)$ & $5(21.7)$ & & $6(22.2)$ & $9(23.7)$ & \\
\hline \multirow[t]{2}{*}{ Tumor Histology } & Adenocarcinoma & $45(93.8)$ & $23(92)$ & 0.78 & $30(96.8)$ & $38(90.5)$ & 0.29 \\
\hline & Other Carcinoma & $3(6.3)$ & $2(9)$ & & $1(3.2)$ & $4(9.5)$ & \\
\hline \multirow[t]{2}{*}{ Surgical Margins } & Negative & $40(83.3)$ & $22(88)$ & 0.60 & $26(83.9)$ & $36(85.7)$ & 0.83 \\
\hline & Positive & $8(16.7)$ & $3(12)$ & & $5(16.1)$ & $6(14.3)$ & \\
\hline \multirow[t]{2}{*}{ Known Post-op CA 19-9 } & $\leq 90$ & $38(82.6)$ & $13(65)$ & 0.12 & $24(85.7)$ & $27(71.1)$ & 0.16 \\
\hline & $>90$ & $8(17.4)$ & $7(35)$ & & $4(14.3)$ & $11(28.9)$ & \\
\hline
\end{tabular}


Table 2: Multivariate analysis of overall survival among all patients and patients who received adjuvant radiation therapy (RT). Among irradiated patients, radioresistant patients (Radiosensitivity Index [RSI] High) had worse survival on Cox multivariate (MV) analysis.

\begin{tabular}{|c|c|c|c|c|c|}
\hline Variable & & UV HR (95\% CI) & UV p-value & MV HR (95\% CI) & MV p-value \\
\hline \multicolumn{6}{|c|}{ All Patients $(n=73)$} \\
\hline \multirow{2}{*}{ Risk } & Low & \multicolumn{2}{|c|}{ Ref } & \multicolumn{2}{|c|}{ Ref } \\
\hline & High & $2.3(1.2,4.4)$ & 0.01 & $2.3(1.2,4.4)$ & 0.01 \\
\hline \multirow{2}{*}{ Adjuvant RT } & No & \multicolumn{2}{|c|}{ Ref } & \multicolumn{2}{|c|}{ Ref } \\
\hline & Yes & $0.9(0.5,1.7)$ & 0.82 & - & $\mathrm{NI}$ \\
\hline \multirow{2}{*}{ RSI } & Low & \multicolumn{2}{|c|}{ Ref } & \multicolumn{2}{|c|}{ Ref } \\
\hline & High & $1.0(0.6,1.7)$ & 0.96 & - & NI \\
\hline \multicolumn{6}{|c|}{ Adjuvant RT (n=48) } \\
\hline \multirow{2}{*}{ Risk } & Low & \multicolumn{2}{|l|}{ Ref } & \multicolumn{2}{|l|}{ Ref } \\
\hline & High & $3.7(1.5,9.0)$ & 0.004 & $4.8(1.9,12.2)$ & 0.001 \\
\hline \multirow{2}{*}{ RSI } & Low & \multicolumn{2}{|l|}{ Ref } & \multicolumn{2}{|l|}{ Ref } \\
\hline & High & $1.3(0.7,2.6)$ & 0.45 & $2.1(1.0,4.3)$ & 0.054 \\
\hline \multirow{4}{*}{$\begin{array}{c}\text { Total RT Dose } \\
\text { (Gy) }\end{array}$} & 45 & \multicolumn{2}{|l|}{ Ref } & \multicolumn{2}{|l|}{ Ref } \\
\hline & $50-50.4$ & $1.0(0.4,2.6)$ & 0.96 & - & NI \\
\hline & $>50.4$ & $1.4(0.4,5.0)$ & 0.60 & - & NI \\
\hline & Unknown & $0.8(0.2,2.9)$ & 0.75 & - & NI \\
\hline
\end{tabular}

Abbreviations: $\mathrm{UV}=$ univariate $; \mathrm{HR}=$ hazard ratio; $\mathrm{CI}=$ confidence interval; $\mathrm{MV}=$ multivariate;

Ref $=$ reference NI $=$ not included 


$$
\mathrm{n}=2241
$$

\section{Excluded \\ Non-Surgical Cases $(n=1323)$}

\section{Excluded}

Non-carcinoma histologies $(\mathrm{n}=36)$

Neoadjuvant therapy $(\mathrm{n}=21)$

Incomplete records $(\mathrm{n}=16)$

Metastatic at presentation $(\mathrm{n}=3)$

Died within 90 days of surgery $(n=2)$

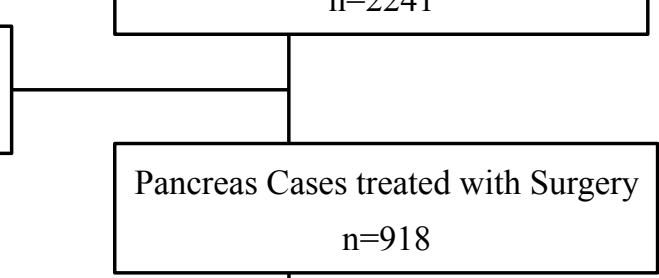

Microarray performed and ranked for RSI

$$
\mathrm{n}=151
$$

Eligible for RSI evaluation

Adjuvant radiation $\mathrm{n}=48$

No adjuvant radiation $n=25$

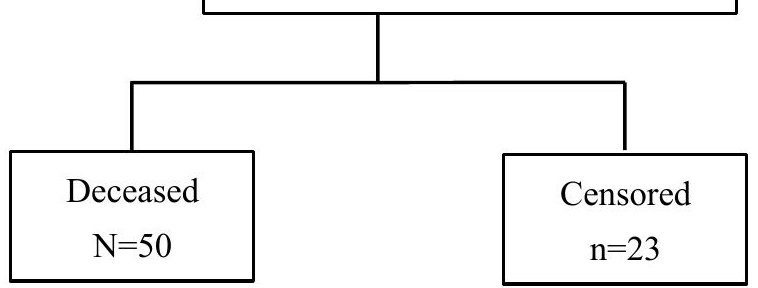




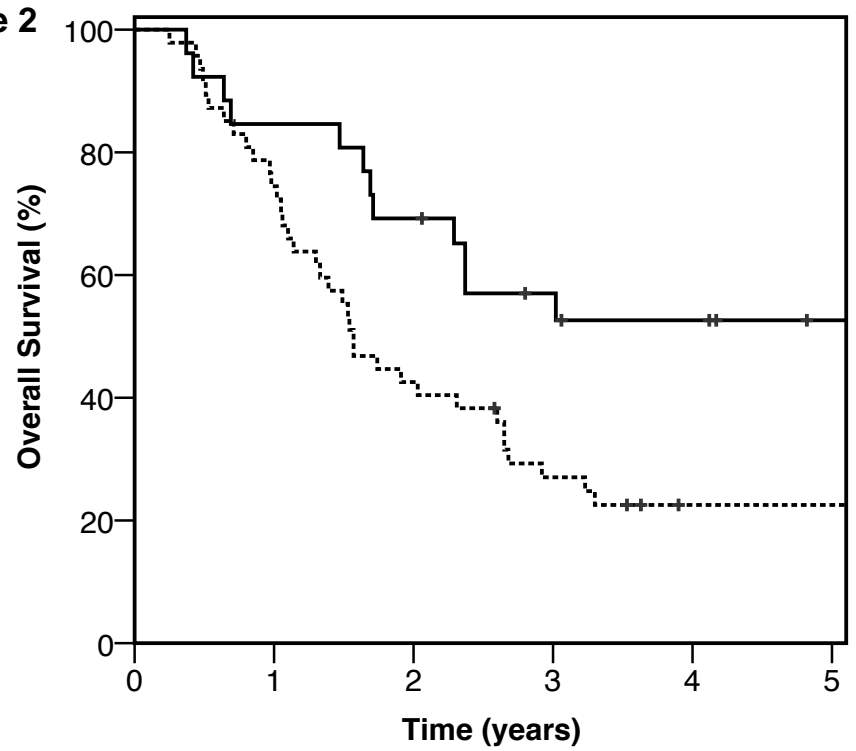

Risk

$\neg$ Low risk -.-.High risk

+ Low risk-censored + High risk-censored

Risk Subjects Events Censored Median Survival (mo, 95\% Cl)

$\begin{array}{lllcc}\text { Low } & 26 & 12 & 14 & \text { Not reached } \\ \text { High } & 47 & 38 & 9 & 18.8(14.9,22.7)\end{array}$

b)

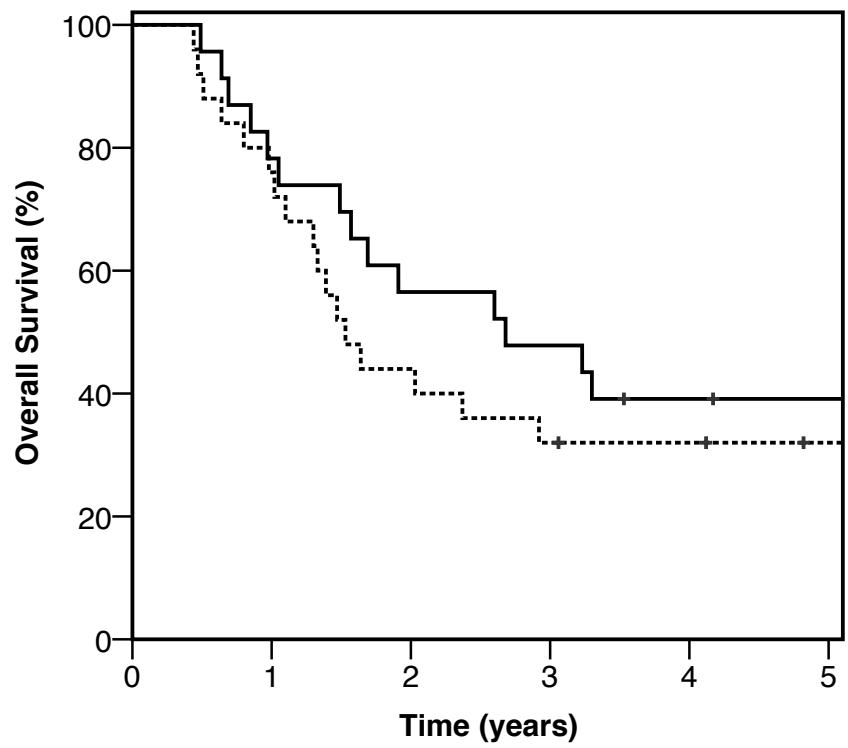

RSI

$\rightarrow \mathrm{PS}$

+ RS-censored

+ RR-censored

RSI

Subjects Events Censored Median Survival $(\mathrm{mo}, 95 \% \mathrm{Cl})$

RS

23

16

7

$32.1(7.3,57.0)$

$\mathrm{RR}$ 
